Canadian

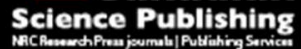

Canadian Journal of Physiology and Pharmacology Revue canadienne de physiologie et pharmacologie

\title{
Digitoxin improves cardiovascular autonomic control in rats with heart failure
}

\begin{tabular}{|r|l|}
\hline Journal: & Canadian Journal of Physiology and Pharmacology \\
\hline Manuscript ID & cjpp-2015-0354.R2 \\
\hline Danuscript Type: & Article \\
\hline Complete List of Authors: & $\begin{array}{l}\text { Fardin, Núbia; University Federal of São Paulo, Physiology } \\
\text { Antonio, Ednei Luiz; Federal University of Sao Paulo } \\
\text { Montemor, Jairo; University Federal of São Paulo, Medicine } \\
\text { da Veiga, Glaucia; University Federal of São Paulo, Physiology } \\
\text { Tucci, Paulo; Universidade Federal de Sao Paulo } \\
\text { Campos, Ruy; University Federal of São Paulo - UNIFESP, Physiology }\end{array}$ \\
\hline Keyword: & $\begin{array}{l}\text { Heart failure, digitoxin, baroreflex sensitivity, renal sympathetic nerve } \\
\text { activity, spectral analysis }\end{array}$ \\
\hline
\end{tabular}


Word count of abstract: 199

Word count of text: 4125

Number of references: 33

Number of figures and tables: 6

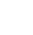

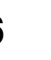

8

9

\section{Digitoxin improves cardiovascular autonomic control in} rats with heart failure

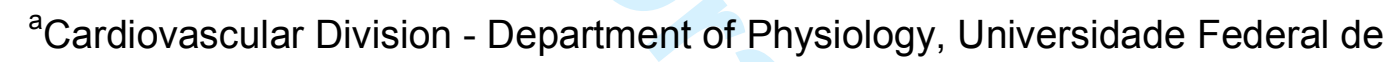

São Paulo - Escola Paulista de Medicina

${ }^{\mathrm{b}}$ Cardiology Division - Department of Medicine, Universidade Federal de São Paulo.

Running Head: Digitoxin improves cardiovascular autonomic control Correspondence: Ruy R. Campos, Ph.D., Cardiovascular Division Department of Physiology, Universidade Federal de São Paulo - Escola Paulista de Medicina. Rua Botucatu, 862, CEP 04023-060, São Paulo, SP, Brazil

$$
\text { Phone: } \quad+55115573-7820 \text { ext.27 }
$$

Fax: $\quad+55115573-7820$ ext.5

24 E-mail: campos@fcr.epm.br 


\section{ABSTRACT}

26 The effects of chronic treatment with digitoxin on arterial baroreceptor sensitivity

27 for heart rate (HR) and renal sympathetic nerve activity (rSNA) control,

28 cardiopulmonary reflex, and autonomic HR control in an animal model of heart

29 failure were evaluated. Wistar rats were treated with digitoxin, which was

30 administered in their daily feed $(1 \mathrm{mg} / \mathrm{Kg} /$ day $)$, for 60 days. The following three

31 experimental groups were evaluated: Sham, heart failure (HF), and HF treated

32 with digitoxin (HF+DIG). We observed an increase in rSNA in the HF group

$33(190 \pm 29 p p s, n=5)$ compared with the Sham group (98 $\pm 14 p p s, n=5)$. Digitoxin

34 treatment prevented an increase in rSNA (98 $\pm 14 \mathrm{pps}, n=7)$. Therefore, arterial

35 baroreceptor sensitivity was decreased in the HF group (-

$361.24 \pm 0.07 \mathrm{bpm} / \mathrm{mmHg}, n=8)$ compared with the Sham group (-

$372.27 \pm 0.23 \mathrm{bpm} / \mathrm{mmHg}, n=6)$. Digitoxin did not alter arterial baroreceptor sensitivity in the HF+DIG group. Finally, the HF group showed an increased low frequency band (LFb: $\left.23 \pm 5 \mathrm{~ms}^{2}, n=8\right)$ and a decreased high frequency band

40 (HFb: $77 \pm 5 \mathrm{~ms}^{2}, n=8$ ) compared with the Sham group (LFb: $14 \pm 3 \mathrm{~ms}^{2}$; HFb:

$4186 \pm 3 \mathrm{~ms}^{2}, n=9$ ); the HF+DIG group exhibited normalized parameters (LFb:

$4215 \pm 3 \mathrm{~ms}^{2}$; HFb: $85 \pm 3 \mathrm{~ms}^{2}, n=9$ ). In conclusion, the benefits of decreasing rSNA

43 are not directly related to improvements in peripheral cardiovascular reflexes;

44 such occurrences are due in part to changes in the central nuclei of the brain

45 responsible for autonomic cardiovascular control.

46 Keywords: Heart failure; digitoxin; baroreflex sensitivity; cardiopulmonary

47 reflex; renal sympathetic nerve activity; spectral analysis; sympathetic

48 vasomotor tone 


\section{INTRODUCTION}

50

51

52

53

54

71 Impairment of arterial baroreceptor function in HF could be an additional factor

72 leading to increases in sympathetic drive (Han et al. 2010) and a cardiac 
73 autonomic imbalance characterized by sympathetic hyperactivity and reduced

74 vagal tone (Morais et al. 2015).

75 One of the pharmacological choices to treat HF is digitalis, which

76 improves systolic and diastolic function, as well as the autonomic balance to the

77 heart ("The effect of digoxin on mortality and morbidity in patients with heart

78 failure. The Digitalis Investigation Group" 1997). Digitalis decreases plasma

79 renin activity and consequently alters the levels of Ang II and aldosterone and

80 decreases serum levels of norepinephrine (Gheorghiade and Ferguson 1991).

81 Digitalis inhibits the $\mathrm{Na}+/ \mathrm{K}+/ \mathrm{ATPase}$ and may promote an increase in arterial

82 baroreceptor sensitivity (Gheorghiade and Ferguson 1991).

83 However, the mechanisms triggering increased sympathetic activity at

84 different targets in HF and how digitalis decreases sympathetic drive in this

85 condition are not well-understood. The present study aimed to investigate

86 changes in renal sympathetic nerve activity and autonomic cardiovascular

87 function, an investigation that included a spectral analysis of blood pressure and

88 arterial baroreceptor and cardiopulmonary reflex sensitivity, in HF rats

89 chronically treated with digitoxin.

90 METHODS

$91 \quad$ All experimental procedures were conducted according to the National

92 Institutes of Health Guidelines for the Use and Care of Animals, and the study

93 protocol was approved by the Ethics in Research Committee of the University

94 Federal of São Paulo (process No. 0442/11). Male Wistar rats (initially weighing

$95200 \mathrm{~g}$ ) were obtained from the animal care facility of our institution. The animals

96 were housed in group cages, given access to rat chow and water ad libitum and 
97 maintained in a temperature-controlled environment $\left(23^{\circ} \mathrm{C}\right)$ on a $12-\mathrm{h}$ light/dark

98 cycle.

\section{EXPERIMENTAL PROTOCOLS}

100 The experiments were performed with the following three experimental

101 groups: Sham, HF and HF+DIG. Seven days after surgery to induce myocardial

102 infarction, the rats were submitted to Doppler echocardiography, and the rats

103 with infarctions involving a minimum of $40 \%$ of left ventricular cardiac mass

104 were selected and divided into two groups as follows: without treatment (HF)

105 and submitted to digitoxin treatment for 60 consecutive days (HF+DIG). At the

106 end of treatment, the mean arterial pressure (MAP), heart rate (HR), baroreflex

107 control of HR and cardiopulmonary reflex were assessed in conscious rats. The

108 spectral analysis of blood pressure was analysed offline (sample rate $2 \mathrm{KHz}$ ).

109 The same group of rats was slowly anesthetized with urethane (1.4 g/kg, i.v.)

110 (Sigma-Aldrich Co, St Louis, MO, USA) to avoid any changes in MAP, and

111 basal renal sympathetic nervous activity (rSNA) and arterial baroreceptor

112 control of rSNA were assessed.

\section{SURGICAL PROCEDURE}

114 After isoflurine-induced anaesthesia (2\%), the animals were immobilized

115 in the supine decubitus position. A left thoracotomy was performed in the fourth

116 intercostal space, and two perpendicularly positioned murine retractors

117 separated the ribs. Radiofrequency current (RF) lesions were created with a

118 commercially available, conventional RF generator (model TEB RF10;

119 Tecnologia Eletrônica Brasileira Ltda, São Paulo, Brazil). RF current (1000

$120 \mathrm{KHz}$ ) was delivered at constant power (12 watts) for 12 seconds. The power

121 output was automatically shut down if the impedance exceeded 200 ohms. The 
122 damaged area was immediately confirmed by the presence of a clear white,

123 disk-shaped region of coagulation necrosis. Thereafter, the heart was quickly

124 returned to the thorax; pulmonary hyperinsufflation was performed, and a

125 previously made purse string suture was used to close the chest. The above

126 procedures were performed according to a previous report (Antonio et al. 2009).

127 DOPPLER ECHOCARDIOGRAPHY AND PULMONARY WATER CONTENT

128 Doppler echocardiographic examinations were performed using an HP

129 SONOS 5500 (Philips Medical System, Andover, MA) with a 12-MHz transducer

130 at a depth of $2 \mathrm{~cm}$ according to a previous report (Antonio et al. 2009; Cury et

131 al. 2005; Kanashiro et al. 2006). Briefly, seven days after surgery, the rats were

132 anesthetized with ketamine + xylazine, and 2-dimensional and M-mode images

133 from the parasternal longitudinal, transverse, and apical views were obtained

134 and recorded on a 0.5 -inch videotape. The image analysis and measurements

135 were performed offline. ECHO detected MI based on subjective identification of

136 akinesis or dyskinesis. The MIS was determined posteriorly in the stained

137 myocardium at the completion of the protocol as described below. The end-

138 diastolic (LVAd) and end-systolic (LVAs) LV transverse areas were measured in

139 the 3 transverse planes (basal, medium, and apical), and LV systolic function

140 was assessed by the change in fractional area (FAC: LVAd - LVAs/LVAd X

141 100). Diastolic function was assessed by calculating the peak $E$ and $A$ blood

142 flow mitral velocities and the E/A ratio. For this purpose, the sample volume of

143 the pulsed wave Doppler was positioned at the tips of the mitral valve leaflets in

144 an apical 4-chamber view.

145 Pulmonary water content (\%) was obtained based on wet and dry

146 weights. After measuring the right lung wet weight, the tissue was placed in an 
147 oven and maintained at $80^{\circ} \mathrm{C}$ for 72 hours. After measuring the dry weight of

148 each rat lung, the water content was determined using the following equation:

149 (wet weight and dry weight/wet weight) $\times 100$.

150 COMPOSITION AND DIET SUPPLEMENTED WITH DIGITOXIN

151 According to a previous report (Helber et al. 2004), the concentration of

152 digitoxin was determined by taking into account the following: (i) orally

153 administered digitoxin is completely absorbed, (ii) a daily dose similar to the one

154 used in the present study was previously shown to be efficient in promoting

155 cardiac benefits in the rat (100 $\mu \mathrm{g} / \mathrm{kg}$ per day) when injected subcutaneously,

156 and (iii) young and adult healthy rats in our laboratory eat approximately $30 \mathrm{~g}$ of

157 food daily. Digitoxin (1 mg) was diluted in absolute ethyl alcohol (3 mL sprayed

158 in and mixed with standard diet $(100 \mathrm{~g})$ ). The resultant chow, with digitoxin

159 added and mixed with water, resulted in dough. Chow pellets were made and

160 left to dry for $24 \mathrm{~h}$ at $50^{\circ} \mathrm{C}$ to allow for the complete evaporation of the alcohol.

161 ANALYSIS OF CARDIOVASCULAR FUNCTION IN CONSCIOUS RATS

162 For the intravenous injection of drugs and direct arterial pressure

163 recording, the rats were anesthetized with ketamine and xylazine (40 and 20

$164 \mathrm{mg} / \mathrm{kg}$, i.p., respectively) (Vetbrands, Jacareí, Brazil) and fitted with femoral

165 venous and arterial catheters. After $\geq 24 \mathrm{~h}$ of surgical recovery, MAP and HR

166 were recorded in conscious rats using an analog-

167 digital board PowerLab (ADInstruments, Australia).

168 For the analysis of the arterial baroreceptor function in conscious rats,

169 bolus injections $(0.1 \mathrm{ml})$ of phenylephrine $(3,5$ and $7 \mu \mathrm{g} / \mathrm{Kg}$, i.v. $)$ or sodium

170 nitroprusside (1, 2 and $3 \mu \mathrm{g} / \mathrm{kg}$, i.v.) (Sigma-Aldrich Co, St Louis, MO, USA)

171 were administered with $\geq 5$-min intervals between doses until blood pressure 
172 returned to baseline. Values of matching MAP variations ( $\triangle$ MAP from 20 to 55

$173 \mathrm{mmHg})$ with reflex heart rate $(\Delta \mathrm{HR})$ responses were separately plotted for each

174 vasoactive drug to create linear regression curves of baroreceptor function for

175 each group, and their slopes (spikes per second per millimetre of $\mathrm{Hg}$ ) were

176 compared to analyse the changes in baroreflex sensitivity.

\section{SPECTRAL ANALYSIS OF HEART RATE AND SYSTOLIC BLOOD 178 PRESSURE}

179 For the spectral analysis of heart rate and systolic pressure variability, 180 beat-by-beat HR and systolic arterial pressure data were obtained in conscious 181 rats for the first $10 \mathrm{~min}$ of the recording period. The spectral power of the 182 various frequency components of HR and systolic pressure were calculated with 183 fast Fourier transform (FFT) algorithm. This analysis requires data collection at 184 equal time intervals. Therefore, heart rate and systolic pressure were calculated 185 every $100 \mathrm{~ms}$ with a cubic spline interpolation $(10 \mathrm{~Hz})$. The interpolated series 186 were divided into half-overlapping sequential sets of 512 data points (51.2 s).

187 The segments were inspected visually; non-stationary data were not analysed.

188 A Hanning window was used to attenuate the side effects. The power intensity 189 was computed with a direct FFT algorithm for discrete time series. The total 190 power in the low frequency band (LFb: $0.2-0.75 \mathrm{~Hz}$ ) and high frequency band 191 (HFb: $0.75-3 \mathrm{~Hz}$ ) was calculated. The LFb/HFb power ratio was calculated and 192 used as an indicator of cardiac sympathovagal balance (Montano et al. 1994).

\section{CARDIAC AUTONOMIC TONE AND INTRINSIC HEART RATE}

194 Tonic sympathetic and vagal influences to the heart were determined by 195 measuring HR changes after the selective pharmacological blockade of cardiac 196 autonomic receptors. The bradycardic response obtained after a $\beta$-adrenergic 
197 receptor blockade with atenolol (1 mg/kg, i.v. - Sigma-Aldrich Co, St Louis, MO,

198 USA) was used to estimate sympathetic tone. The tachycardia response after 199 muscarinic cholinergic receptor blockade with methyl atropine (3 mg/kg, i.v. -

200 Sigma-Aldrich Co, St Louis, MO, USA) was used to estimate vagal tone. The

$201 \mathrm{HR}$ in the presence of both autonomic blockers was considered the intrinsic HR.

202

203

204

205

206

207

208

209

210

211

212

213

214

215

216

217

218

219

220

221

\section{ANALYSIS OF THE BEZOLD-JARISCH REFLEX SENSITIVITY}

In conscious rats, bolus injections $(0.1 \mathrm{ml})$ of phenylbiguanide (PBN)

$(12.5,25,50 \mu \mathrm{g} / \mathrm{Kg})$ were administered with $\geq 15$-min intervals between doses until blood pressure returned to baseline. The percentages of MAP variation $(\% \Delta \mathrm{MAP})$ and $\mathrm{HR}$ variation $(\% \Delta \mathrm{HR})$ were compared among the groups.

\section{ANALYSIS OF RENAL SYMPATHETIC NERVE ACTIVITY IN URETHANE} ANESTHETIZED RATS

Rats were slowly anesthetized with urethane, and the left renal nerve was retroperitoneally exposed and placed on bipolar silver electrodes. When the conditions for nerve recording were established, the nerve and electrode were covered with paraffin oil. The signal from the renal nerve was displayed on an oscilloscope (Tektronix, TDS 220), and nerve activity was amplified (gain 20000, Neurolog, Digitimer, UK), filtered by a band-pass filter (100-1000 Hz), and collected for display and subsequent analysis using a PowerLab data acquisition system (ADInstruments, Australia). At the end of the experiments, the background noise level was determined following hexamethonium bromide (30 mg/kg, i.v.) (Sigma-Aldrich Co, St Louis, MO, USA) administration. The rSNA was rectified online, integrated from the raw data obtained for each heart period, and expressed as volts-seconds (V.s). Additionally, neural activity was analysed offline using the appropriate software (Spike Histogram, 
222 ADInstruments - Australia). The responses of rSNA to the various stimuli are

223 expressed as the percentage of change compared with the basal value

224 obtained immediately before each test. For this purpose, the raw nerve signal

225 was passed through a spike discriminator (PowerLab) to remove background

226 noise, and the total nerve activity, which is expressed in spikes per second

227 (spikes/s), was computed from the time at which the value changed from the

228 basal value to when it returned to the basal value. The basal rSNA is expressed

229 as spikes/s over a period of $60 \mathrm{~s}$. The mean value obtained was compared with

230 the mean value determined before each test as reported previously. Only

231 experiments in which the level of background noise was confirmed at the end of

232 the experiments following hexamethonium and terminal anaesthesia are

233 included in this report.

234 For the analysis of arterial baroreceptor function sensitivity for rSNA

235 control in anesthetized rats, continuous infusions of phenylephrine $(10 \mu \mathrm{g} / \mathrm{Kg}$,

$2360.2 \mathrm{~mL}, 12 \mathrm{~mL} / \mathrm{h}$, i.v. $)$ or sodium nitroprusside $(20 \mu \mathrm{g} / \mathrm{kg}, 0.1 \mathrm{~mL}, 6 \mathrm{~mL} / \mathrm{h}$, i.v. $)$

237 (Sigma-Aldrich Co, St Louis, MO, USA) were administered with a $1 \mathrm{ml}$ syringe

238 mounted on a syringe pump (model KDS 100, KD Scientific). Values of

239 matching MAP variations ( $\triangle$ MAP from 20 to $55 \mathrm{mmHg}$ ) with reflex renal

240 sympathetic nerve activity $(\triangle \mathrm{rSNA})$ responses were separately plotted for each

241 vasoactive drug to create linear regression curves of baroreceptor function for

242 each group, and their slopes (spikes per second per millimetre of $\mathrm{Hg}$ ) were

243 compared to analyse the changes in baroreflex sensitivity.

244 STATISTICAL ANALYSES

245 The results are shown as the mean \pm standard error of the mean for

246 functional experiments, and the data of the spectral analyses are shown as the 
247 mean \pm standard deviation of the mean. Data were evaluated by one-way

248 ANOVA, followed by the Tukey test. The level of statistical significance was 249 defined as $p<0.05$.

250 RESULTS

251

252

DOPPLER ECHOCARDIOGRAPHY AND PULMONARY WATER CONTENT

253

Table 1 shows the echocardiogram data, the other parameters of cardiac remodelling and the pulmonary water content. All rats used in this study had an 254 area of infarction greater than $40 \%$. The HF group showed an increase in systolic area $\left(0.30 \mathrm{~cm}^{2}, n=9\right)$ and systolic circumference $(1.81 \mathrm{~cm}, n=9)$ compared with the Sham group (systolic area: $0.07 \mathrm{~cm}^{2}$; systolic circumference: $0.86 \mathrm{~cm}, n=5)$. In this case, treatment with digitoxin did not improve these parameters (systolic area: $0.41 \mathrm{~cm}^{2}$; systolic circumference: $2.18 \mathrm{~cm}, n=5$ ). $\mathrm{HF}$ $(80.8 \pm 0.2 \%, n=9)$ and the HF+DIG group $(80.6 \pm 0.1 \%, n=5)$ presented with 260 an increase in pulmonary water content compared with the Sham group $(78.1 \pm$ $0.3 \%, n=5)$.

262 265 266

267 268 269 270

\section{CARDIOVASCULAR AND AUTONOMIC BASAL PARAMETERS}

The HF rats showed decreased MAP $(91 \pm 5.0 \mathrm{mmHg}, n=5)$ and HR (314 $\pm 9.8 \mathrm{bpm}, n=8$ ) compared with the Sham group (MAP: $109 \pm 3 \mathrm{mmHg}, n=5$; HR: $350 \pm 12.7 \mathrm{bpm}, n=6$ ). Digitoxin treatment did not change these values in the HF+DIG group (MAP: $92 \pm 2.1 \mathrm{mmHg}, n=7 ; \mathrm{HR}: 296 \pm 7 \mathrm{bpm}, n=9$ ), as shown in Figure 1A and B. The HF rats presented with increased rSNA (190 \pm $30 \mathrm{pps}, n=5)$ compared with the Sham rats (98 $\pm 14.7 \mathrm{pps}, n=5)$. Digitoxin treatment significantly decreased and normalized rSNA in the HF+DIG group (98 \pm 14.3 pps, $n=7)$, as shown in Figure 1C. Figure 1D shows a representative 
271 tracing of the cardiovascular and autonomic parameters in the Sham, HF and

272 HF+DIG groups.

273 EFFECT OF TREATMENT HEART RATE AND SYSTOLIC PRESSURE 274 VARIABILITY

275 A spectral analysis of HR variability (HRV) showed an increase in LFb in 276 the HF group $\left(23 \pm 5 \mathrm{~ms}^{2}, n=8\right)$ compared with the Sham group $\left(14 \pm 3 \mathrm{~ms}^{2}\right.$, $277 n=9$ ); treatment with digitoxin normalized this parameter in the HF+DIG group $278\left(15 \pm 3 \mathrm{~ms}^{2}, n=9\right)$. The HF group also showed decreased HFb $\left(77 \pm 5 \mathrm{~ms}^{2}, n=8\right)$ 279 compared with the Sham group $\left(86 \pm 3 \mathrm{~ms}^{2}, n=9\right)$; digitoxin normalized this 280 parameter in the HF+DIG group $\left(85 \pm 3 \mathrm{~ms}^{2}, n=9\right)$. The results are summarized 281 in Table 2.

282 A spectral analysis of systolic blood pressure variability showed an 283 increase in LFb in the HF group $\left(6 \pm 1 \mathrm{mmHg}^{2}, n=8\right)$ compared with the Sham 284 group ( $\left.5 \pm 2 \mathrm{mmHg}^{2}, n=9\right)$; digitoxin treatment decreased this parameter in the 285 HF+DIG group $\left(2 \pm 0.3 \mathrm{mmHg}^{2}, n=9\right)$. The HF+DIG group also showed a 286 decrease in HFb $\left(1 \pm 0.3 \mathrm{mmHg}^{2}, n=9\right)$ compared to HF group $\left(3 \pm 0.4 \mathrm{mmHg}^{2}\right.$, $287 n=8)$ and Sham group $\left(2 \pm 0.4 \mathrm{mmHg}^{2}, n=9\right)$. The results are summarized in 288 Table 2.

289 ARTERIAL BAROREFLEX SENSITIVITY:

290 A preferential decrease in baroreflex sensitivity for HR control induced by 291 phenylephrine (baroreceptor loading) was observed in the HF and HF+DIG 292 groups compared with the Sham group, as shown in Figure 2 A, and the 293 individual slope values are shown in Table 3. However, no difference among 294 the groups was found in relation to baroreceptor sensitivity for rSNA control, as 295 shown in Figure 2 B, and the individual slope values are shown in Table 3. 
297 BEZOLD-JARISCH REFLEX:

298 The HF and HF+DIG groups showed a decrease in \% $\triangle M A P$ responses in 299 all doses of PBN (HF: $12.5 \mu \mathrm{g}:-17.2 \pm 3.6 \mathrm{mmHg} ; 25 \mu \mathrm{g}:-27.6 \pm 3.8 \mathrm{mmHg} ; 50$

$300 \mu \mathrm{g}:-30.3 \pm 3.4 \mathrm{mmHg}, n=11$; HF+DIG: $25 \mu \mathrm{g}:-26.4 \pm 4.8 \mathrm{mmHg}, n=12)$

301 compared with the Sham group $(12.5 \mu \mathrm{g}:-34.5 \pm 3 \mathrm{mmHg}$; $25 \mu \mathrm{g}$ : $-47.4 \pm 5.6$

$302 \mathrm{mmHg} ; 50 \mu \mathrm{g}:-53.3 \pm 4 \mathrm{mmHg}, n=7$ ), as shown in Figure $3 \mathrm{~A}$. The HF group

303 also showed a decrease in $\% \Delta H R$ at intermediate and highest doses of PBN

304 (25 $\mu \mathrm{g}:-52.2 \pm 3 \mathrm{bpm}, n=8$ and $50 \mu \mathrm{g}:-56.3 \pm 2.5 \mathrm{bpm}, n=8)$ compared with the

305 Sham group ( $25 \mu \mathrm{g}:-71 \pm 2.5 \mathrm{bpm}, n=4$ and $50 \mu \mathrm{g}:-72.5 \pm 2.2 \mathrm{bpm}, n=4)$, and

306 digitoxin treatment $(\mathrm{HF}+\mathrm{DIG})$ promoted a decrease in $\% \Delta \mathrm{HR}$ for the same

307 doses of PBN ( $25 \mu \mathrm{g}:-40.4 \pm 2.8 \mathrm{bpm}, n=9$ and $50 \mu \mathrm{g}:-40 \pm 3.7 \mathrm{bpm}, n=9)$

308 compared with the HF group, as shown in Figure $\mathbf{3}$ B.

309

310 DISCUSSION

311 The major new findings of the present study were as follows: 1) digitoxin

312 treatment prevented increases in rSNA and alterations of the autonomic

313 balance to the heart and blood vessels in the HF-treated group (HF+DIG) and

314 2) these improvements in cardiovascular function were independent of cardiac

315 remodelling and peripheral cardiovascular reflexes, including the arterial

316 baroreceptor and cardiopulmonary reflexes.

317 The mechanisms underlying sympathoexcitation in HF are not well-

318 understood. Some hypotheses suggest that reflex sympathoexcitation is a

319 consequence of decreased blood pressure and increased circulating and tissue 
320 Ang II (DiBona et al. 1995) or that sympathoexcitation occurs even in response

321 to a decrease in arterial baroreflex sensitivity. These processes may result in a

322 reduction in the inhibitory influence on afferent vasomotor centres in the brain,

323 resulting in neuro-humoral efferent sympathetic outflow (Ferguson et al. 1984b).

324 Considering that increased sympathetic activity to the kidneys leads to

325 important changes in kidney function, including renin secretion, vasoconstriction

326 and sodium reabsorption (DiBona et al. 1995) and that all of these changes are

327 present in HF, new strategies to control autonomic dysfunction in HF are

328 important for the outcome of the disease.

329 In the present study, we observed a preferential decrease in

330 baroreceptor reflex sensitivity for HR but not for rSNA in the HF rats compared

331 with the Sham group. These data suggest that the increased rSNA in the HF

332 group was not directly related to changes in baroreceptor reflex sensitivity, as

333 previously reported (Ferguson et al. 1984a).

334 The decrease in MAP in the HF rats could trigger other compensatory

335 mechanisms, such as renin-angiotensin-aldosterone system activation, which

336 could contribute to increases in rSNA, as proposed previously (Dibona et al.

337 1995). However, in the present study, a significant decrease in rSNA was found

338 in the HF+DIG group, suggesting that sympathoexcitation is not related to low

339 blood pressure because the HF+DIG and HF groups had similar blood pressure

340 levels. Changes in the central nuclei in the brain related to cardiovascular

341 control could be involved in the increases in rSNA; we also noted an imbalance

342 in the autonomic control of the heart with an increase in LFb and a decrease in

343 HFb in the HF rats. Wang et al. (Wang et al. 2009) found that microinjections of

344 a glutamate receptor antagonist into the rostralventrolateral medulla (RVLM) of 
345 HF rats significantly decreased MAP and rSNA, suggesting that increased

346 glutamate receptor expression in the RVLM contributes to increased

347 sympathetic vasomotor drive in HF.

348 However, there is controversy regarding baroreceptor dysfunction in HF.

349 The divergence is related to the animal model used for HF induction, the

350 extension of myocardial infarction and the time after myocardial infarction at

351 which the experiments were performed. According to Ramchandra et al.

352 (Ramchandra et al. 2014), baroreflex control for HR and rSNA, but not for

353 cardiac sympathetic nerve activity (cSNA), was impaired in sheep with HF.

354 However, Zucker et al. (Zucker et al. 1985) reported that arterial baroreceptor

355 sensitivity for rSNA in dogs with HF was normal compared with control animals.

356 This controversy is not limited only to HF animal models. Floras et al. (Floras,

357 2001) found that humans with advanced HF had decreased baroreceptor reflex

358 sensitivity relative to HR control but not to muscle ANS compared with healthy

359 subjects. Others (Dibnerdunlap and Thames 1989; Grassi et al. 1995) noted

360 decreased sensitivity of baroreceptor reflex responses to muscle sympathetic

361 nerve activity in humans with HF.

362 Together, these studies suggest a territory-dependent difference in

363 baroreceptor reflex sensitivity in HF in different species. The same was found in

364 the present study: a decrease was noted in the sensitivity of the baroreceptor

365 reflex for HR control but not for of rSNA control.

366 However, this was the first study to investigate baroreceptor function in a

367 model of HF induced by ablation. The ablation model of HF was chosen due to

368 its low mortality rates and its low variability in the size of the infarct area among

369 animals. In addition, this model does not cause papillary muscle injury, as noted 
370 in the occlusion model, and thus does not lead to changes in cardiac valves that

371 could hamper the interpretation of the results. In addition, as presented in Table

3721 (echocardiography parameters), this model has specific characteristics of HF,

373 as described in other models of HF animals (dos Santos et al. 2008; Moisés et

374 al. 2000).

375 We noted significant impairment of the cardiopulmonary reflex in $\mathrm{HF}$

376 animals; a similar finding was previously reported (Modesti et al. 2004).

377 Ferguson et al. (Ferguson et al. 1984b) found that the decreased sensitivity of

378 the cardiopulmonary and arterial baroreceptor reflexes may result in decreased

379 afferent inhibitory influence on the vasomotor centres of the brain, resulting in

380 increased sympathetic efferent and neurohumoral excitation. Thus, we can infer

381 that the reduction in the sensitivity of the cardiopulmonary reflex is one of the

382 factors that may trigger increased rSNA. However, digitoxin treatment

383 significantly reduced rSNA and caused further impairment of the

384 cardiopulmonary reflex, suggesting that rSNA reduction induced by digitoxin in

385 HF rats is not directly related to the cardiopulmonary reflex.

386 Digitoxin sensitizes the vagal afferents mediating the cardiopulmonary

387 reflex, leading to a reduction in rSNA in dogs (Thames et al. 1982). These

388 findings could have implications concerning the potential mechanism of action

389 of digitalis in heart failure. However, in the present study, there was no

390 correlation between the sympathoinhibition induced by digitoxin and the

391 cardiopulmonary reflex. Therefore, the improvement in survival induced by

392 digitoxin in HF rats could be related to rSNA reduction (Helber and Tucci 2010).

393 Another interesting result was that the animals treated with digitoxin

394 showed no improvement in cardiac remodelling, as shown by the 
395 echocardiographic data in Table 1. However, there was improvement and

396 normalization of autonomic balance to the heart and blood vessels, as shown

397 by the HRV results in Table 2. These results demonstrate that digitoxin has

398 cardioprotective effects and promotes the reduction of sympathetic vasomotor

399 drive. Moreover, we believe that digitalis influences autonomic modulation, most

400 likely through central actions in the brain. In addition, digitoxin may act directly

401 on the sinus node, improving the autonomic balance to the heart because

402 digitoxin acts on the $\mathrm{Na}^{+} / \mathrm{K}^{+} / \mathrm{ATPase}$, which is present in all cells. In excitable

403 tissues, its activity is essential for regulating membrane potential and is

404 responsible for cellular excitability (Teruya et al. 1997). However, the relative

405 importance of the central versus peripheral actions of digitoxin is still a matter of 406 discussion.

$407 \quad$ Although digitoxin has a very low therapeutic range and can be extremely 408 toxic, we used a study previously conducted in our laboratory as a reference 409 (Helber, et al. 2004) as this study described the importance of serum digitoxin 410 and the mortality rates of infarcted animals. Furthermore, according

411 Wasserstrom and Aistrup (2005), rats are less sensitive to cardiac glycoside

412 due to resistance in alpha isoform 1; therefore, the dose used in this study was

413 higher than that used in humans but was not toxic to the animals (Helber, et al. 414 2004).

$415 \quad$ Conclusion:

416 In the present study, digitoxin reduced and normalized rSNA and

417 improved the autonomic balance to the heart in HF rats; similar data have been

418 previously described (Gheorghiade and Ferguson 1991; Mason and Braunwald

419 1964). However, the mechanism underlying the sympathoinhibition induced by 
420 digitoxin remains unknown. Because the HF animals treated with digitoxin

421 showed no improvement in the sensitivities of their arterial and cardiopulmonary

422 baroreceptor reflexes, it is possible that digitoxin acts in the brain to reduce

423 sympathetic vasomotor drive to the kidneys and heart.

424

425

426

\section{ACKNOWLEDGEMENTS}

427

This work was supported by grants from CAPES and FAPESP

428 (2009/54225-8). Ruy R Campos and Paulo Tucci are recipients of CNPq

429 fellowships.

430

431 DISCLOSURE

$432 \quad$ The authors declare no conflicts of interest.

433

434 References

435 Antonio, E. L., Dos Santos, A. A., Araujo, S. R., Bocalini, D. S., Dos Santos, L., 436 Fenelon, G., et al. 2009. Left ventricle radio-frequency ablation in the rat: a new 437 model of heart failure due to myocardial infarction homogeneous in size and low 438 in mortality. J. Card. Fail. 15(6): 540-548.

439 Booth, L. C., Schlaich, M. P., Nishi, E. E., Yao, S. T., Xu, J., Ramchandra, R., et 440 al. 2015. Short-term effects of catheter-based renal denervation on cardiac 441 sympathetic drive and cardiac baroreflex function in heart failure. Int. J. Cardiol. 442 190: 220-226.

443 Carillo, B. A., Oliveira-Sales, E. B., Andersen, M., Tufik, S., Hipolide, D., 444 Santos, A. A., et al. 2012. Changes in GABAergic inputs in the paraventricular 445 nucleus maintain sympathetic vasomotor tone in chronic heart failure. Auton. 446 Neurosci. 171(1-2): 41-48.

447 Coons, J. C., McGraw, M., and Murali, S. 2011. Pharmacotherapy for acute 448 heart failure syndromes. Am. J. Health. Syst. Pharm. 68(1): 21-35. 
449 Cury, A. F., Bonilha, A., Saraiva, R., Campos, O., Carvalho, A. C., De Paola, A. 450 A., et al. 2005. Myocardial performance index in female rats with myocardial 451 infarction: relationship with ventricular function parameters by Doppler 452 echocardiography. J. Am. Soc. Echocardiogr. 18(5): 454-460.

453 Dibnerdunlap, M. E., and Thames, M. D. 1989. Baroreflex control of renal 454 sympathetic nerve activity is preserved in heart failure despite reduced arterial 455 baroreceptor sensitivity. Circ. Res. 65(6): 1526-1535.

456 DiBona, G. F., Jones, S. Y., and Brooks, V. L. 1995. ANG II receptor blockade 457 and arterial baroreflex regulation of renal nerve activity in cardiac failure. Am. J. 458 Physiol. 269(5 Pt 2): R1189-1196.

459 Dibona, G. F., Jones, S. Y., and Brooks, V. L. 1995. ANG-II receptor blockade 460 and arterial baroreflex regulation of renal nerve activity in cardiac failure. Am. J. 461 Physiol. 269(5): R1189-R1196.

462 dos Santos, L., Mello, A. F. S., Antonio, E. L., and Tucci, P. J. F. 2008.

463 Determination of myocardial infarction size in rats by echocardiography and 464 tetrazolium staining: correlation, agreements, and simplifications. Brazilian J. of 465 Med. Biol. Res. 41(3): 199-201.

466 Ferguson, D. W., Abboud, F. M., and Mark, A. L. 1984a. Selective impairment 467 of baroreflex-mediated vasoconstrictor responses in patients with ventricular 468 dysfunction. Circulation, 69(3): 451-460.

469 Ferguson, D. W., Abboud, F. M., and Mark, A. L. 1984b. Selective impairment 470 of baroreflex-mediated vasoconstrictor responses in patients with ventricular 471 dysfunction. Circulation, 69(3): 451-460.

472 Floras, J. S. 2001. Arterial baroreceptor and cardiopulmonary reflex control of 473 sympathetic outflow in human heart failure. Neuro-Cardiovascular Regulation: 474 from Molecules to Man, 940: 500-513.

475 Gao, L., Wang, W. Z., Wang, W., and Zucker, I. H. 2008. Imbalance of 476 angiotensin type 1 receptor and angiotensin II type 2 receptor in the rostral 477 ventrolateral medulla: potential mechanism for sympathetic overactivity in heart 478 failure. Hypertension, 52(4): 708-714.

479 Gheorghiade, M., and Ferguson, D. 1991. Digoxin. A neurohormonal modulator 480 in heart failure? Circulation, 84(5): 2181-2186.

481 Grassi, G., Seravalle, G., Cattaneo, B. M., Lanfranchi, A., Vailati, S., 482 Giannattasio, C., et al. 1995. Sympathetic activation and loss of reflex 483 sympathetic control in mild congestive-heart-failure. Circulation, 92(11): 32064843211.

485 Hamdani, N., and Paulus, W. J. Treatment of heart failure with normal ejection 486 fraction. 2011. Curr. Treat. Options Cardiovasc. Med. 13(1): 26-34. 
487 Han, T. H., Lee, K., Park, J. B., Ahn, D., Park, J. H., Kim, D. Y., et al. 2010.

488 Reduction in synaptic GABA release contributes to target-selective elevation of 489 PVN neuronal activity in rats with myocardial infarction. Am. J. Physiol. 299(1):

490 R129-139.

491 Helber, I., and Tucci, P. J. 2010. [Digoxin: the results of the DIG study in the 492 XXI century]. Arq. Bras. Cardiol. 95(4): e108-111.

493 Helber, I., Kanashiro, R. M., Alarcon, E. A., Jr., Antonio, E. L., and Tucci, P. J. 494 2004. A novel inexpensive murine model of oral chronic digitalization. Clin. Exp. 495 Pharmacol. Physiol. 31(5-6): 365-366.

496 Kanashiro, R. M., Saraiva, R. M., Alberta, A., Antonio, E. L., Moises, V. A., and 497 Tucci, P. J. 2006. Immediate functional effects of left ventricular reduction: a 498 Doppler echocardiographic study in the rat. J. Card. Fail. 12(2): 163-169.

499 Mangini, S., Silveira, F. S., Silva, C. P., Grativvol, P. S., Seguro, L. F., Ferreira, 500 S. M., et al. 2008. Decompensated heart failure in the emergency department of 501 a cardiology hospital. Arq. Bras. Cardiol. 90(6): 400-406.

502 Mason, D. T., and Braunwald, E. 1964. Studies on digitaliz .X. efects of ouabain 503 on forearm vascular resistance + venous tone in normal subjects + in patients in 504 heart failure. Journal of Clinical Investigation. J. Clin. Invest. 43(3): 532-and.

505 Modesti, P. A., Polidori, G., Bertolozzi, I., Vanni, S., and Cecioni, I. 2004. 506 Impairment of cardiopulmonary receptor sensitivity in the early phase of heart 507 failure. Heart, 90(1): 30-36.

508 Moisés, V. A., Ferreira, R. L., Nozawa, E., Kanashiro, R. M., Campos, O., 509 Andrade, J. L., et al. 2000. Structural and functional characteristics of rat hearts 510 with and without myocardial infarct. Initial experience with Doppler 511 echocardiography. Arq. Bras. Cardiol. 75(2): 125-136.

512 Montano, N., Ruscone, T. G., Porta, A., Lombardi, F., Pagani, M., and Malliani, 513 A. 1994. Power spectrum analysis of heart rate variability to assess the 514 changes in sympathovagal balance during graded orthostatic tilt. Circulation, 515 90(4): 1826-1831.

516 Morais, S. D., Silva, L. E., Lataro, R. M., Silva, C. A., Oliveira, L. F., Carvalho, 517 E. E., et al. 2015. Mesenchymal Stem Cells Improve Heart Rate Variability and 518 Baroreflex Sensitivity in Rats with Chronic Heart Failure. Stem Cells Dev.

519 Parati, G., and Esler, M. 2012. The human sympathetic nervous system: its 520 relevance in hypertension and heart failure. Eur. Heart. J. 33(9): 1058-1066.

521 Ramchandra, R., Hood, S. G., and May, C. N. 2014. Central exogenous nitric 522 oxide decreases cardiac sympathetic drive and improves baroreflex control of 523 heart rate in ovine heart failure. Am. J. Physiol. 307(3): R271-R280. 
524 Teruya, H., Yamazato, M., Muratani, H., Sakima, A., Takishita, S., Terano, Y., 525 et al. 1997. Role of ouabain-like compound in the rostral ventrolateral medulla in 526 rats. J. Clin. Invest. 99(11): 2791-2798.

527 Thames, M. D., Miller, B. D., and Abboud, F. M. 1982. Sensitization of vagal 528 cardiopulmonary baroreflex by chronic digoxin. Am. J. Physiol. 243(5): H815$529 \mathrm{H} 818$.

530 The effect of digoxin on mortality and morbidity in patients with heart failure. 531 The Digitalis Investigation Group 1997. N. Engl. J. Med. 336(8): 525-533.

532 Wang, W. Z., Gao, L., Wang, H. J., Zucker, I. H., and Wang, W. 2009. Tonic 533 Glutamatergic Input in the Rostral Ventrolateral Medulla Is Increased in Rats 534 With Chronic Heart Failure. Hypertension, 53(2): 370-374.

535 Wasserstrom, J. A.; Aistrup, G. L. 2005. Digitalis: new actions for an old drug. 536 Am. J. Physiol. 289 (5): H1781-H1793.

537 Zucker, I. H., Gorman, A. J., Cornish, K. G., and Lang, M. 1985. Impaired atrial 538 receptor modulation or renal nerve activity in dogs with chronic volume 539 overload. Cardiovasc. Res. 19(7): 411-418.

540 


\section{FIGURE LEGENDS}

\section{2}

543

Table 1. Doppler echocardiographic parameters for the Sham, Heart

544 Failure (HF) and HF treated with digitoxin (HF+DIG) groups. Values are the

545 mean \pm standard error of the mean. ${ }^{*} p<0.05$ compared with the Sham group.

546 One-way ANOVA followed by Tukey post-test.

Figure 1. (A) Mean Arterial Pressure (MAP). (B) Heart Rate (HR). (C)

549 renal Sympathetic Nerve Activity (rSNA). (D) Typical tracings of MAP, HR and 550 rSNA. Sham and Heart Failure (HF) untreated and treated (HF+DIG). Values

551 are the mean \pm standard error of the mean. ${ }^{*} p<0.05$ compared with the Sham 552 group; \#p<0.05 compared with the Heart Failure (HF) group. One-way ANOVA 553 followed by Tukey post-test.

Table 2. Blood pressure and pulse interval variability for the Sham, Heart

556 Failure (HF) and HF treated with digitoxin (HF+DIG) groups. Values were

557 compared and are presented as the mean \pm standard deviation. ANOVA test

558 followed by Tukey post-test. * $p<0.05$ vs Sham and $\# p<0.05$ vs HF.

559 Abbreviations: $\mathrm{LFb}=$ Low frequency band, HFb = High frequency band.

560

561 Figure 2. (A) Baroreceptor reflex function for heart rate (HR) control.

562 Linear regression graph containing (1) reflex increases in HR in response to 563 decreases in mean arterial pressure (MAP) produced by bolus injections of $(0.1$

$564 \mathrm{ml}$ ) sodium nitroprusside (1, 2 and $3 \mu \mathrm{g} / \mathrm{kg}$, i.v.) and (2) reflex decreases in HR 565 in response to increased MAP produced by bolus injections of $(0.1 \mathrm{ml})$ 
566 phenylephrine (3, 5 and $7 \mu \mathrm{g} / \mathrm{Kg}$, i.v.). (B) Baroreceptor reflex function for renal

567 sympathetic nerve activity (rSNA) control. Nonlinear regression graph

568 containing (1) reflex increases in rSNA in response to decreases in mean

569 arterial pressure (MAP) produced by continuous infusions of sodium

570 nitroprusside (20 $\mathrm{gg} / \mathrm{kg}, 0.1 \mathrm{~mL}, 6 \mathrm{~mL} / \mathrm{h}$, i.v. $)$ and (2) reflex decreases in rSNA in

571 response to increased MAP produced by continuous infusions of phenylephrine

$572(10 \mu \mathrm{g} / \mathrm{Kg}, 0.2 \mathrm{~mL}, 12 \mathrm{~mL} / \mathrm{h}$, i.v. $)$. Values are the mean \pm standard error of the

573 mean. * $p<0.05$ compared with the Sham group before digitoxin; and $\# p<0.05$

574 compared with the Heart Failure (HF) group before digitoxin. ${ }^{*} p<0.05$ compared

575 with the Sham group. One-way ANOVA followed by Tukey post-test.

576

577 Table 3. Slope values obtained from the linear and nonlinear regression

578 lines of the baroreceptor reflex function control for heart rate (HR) and of the

579 renal sympathetic nerve activity (rSNA), respectively, for the Sham, Heart

580 Failure (HF) and HF treated with digitoxin (HF+DIG) groups. Values are the

581 mean \pm standard error of the mean. ${ }^{*} p<0.05$ compared with the Sham group.

582 One-way ANOVA followed by Tukey post-test.

583

584 Figure 3. Bezold-Jarisch reflex. (A) Percentage of mean arterial pressure 585 variation $(\% \Delta M A P)$ induced by bolus injection $(0.1 \mathrm{ml})$ of phenylbiguanide $(\mathrm{PBN})$

$586(12.5,25,50 \mu \mathrm{g} / \mathrm{Kg})$. (B) Percentage of heart rate variation (\% $\% \mathrm{HR})$ induced by

587 bolus injection $(0.1 \mathrm{ml})$ of phenylbiguanide $(\mathrm{PBN})(12.5,25,50 \mu \mathrm{g} / \mathrm{Kg})$. Values

588 are the mean \pm standard error of the mean. ${ }^{*} p<0.05$ compared with the same

589 dose of phenylbiguanide (PBN) in the Sham group; $\# p<0.05$ compared with the 
590 same dose of PBN in the Heart Failure (HF) group. One-way ANOVA followed

591 by Tukey post-test.

592 
A

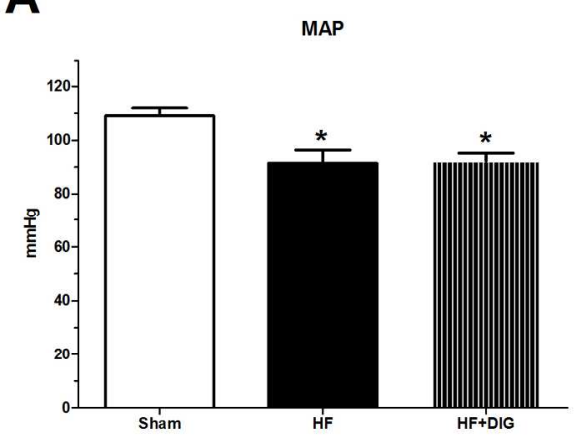

B

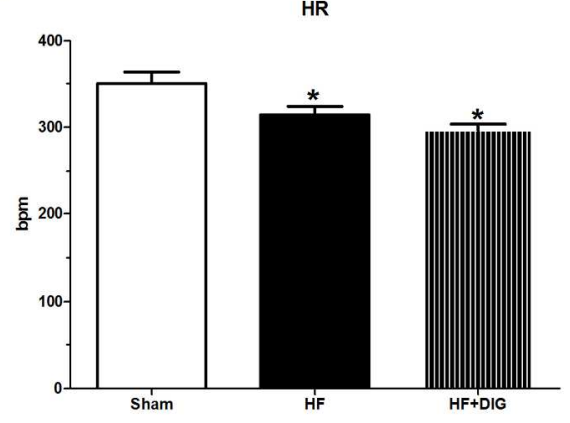

C

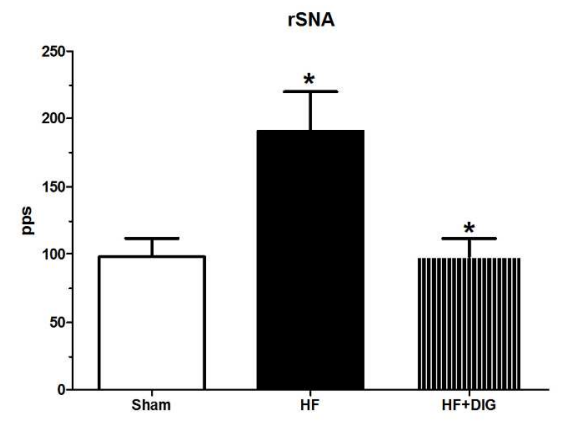

D

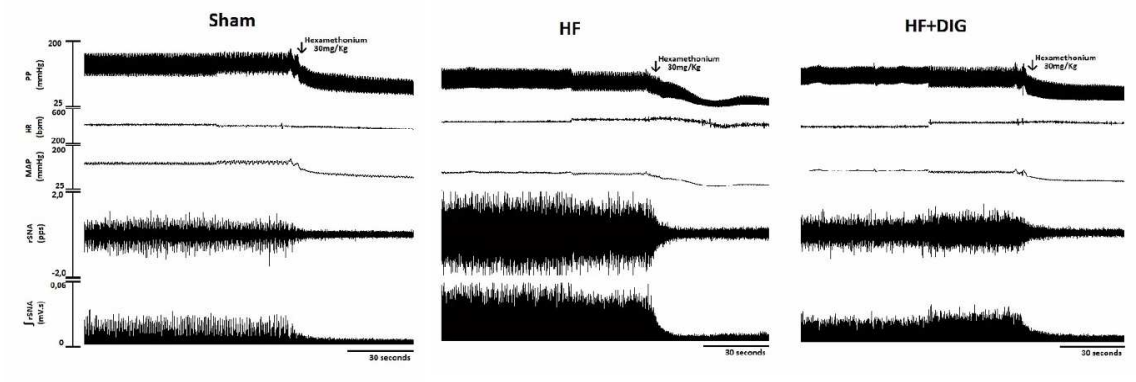

$183 \times 213 \mathrm{~mm}(300 \times 300 \mathrm{DPI})$ 
A

Baroreflex sensitivity of heart rate

1

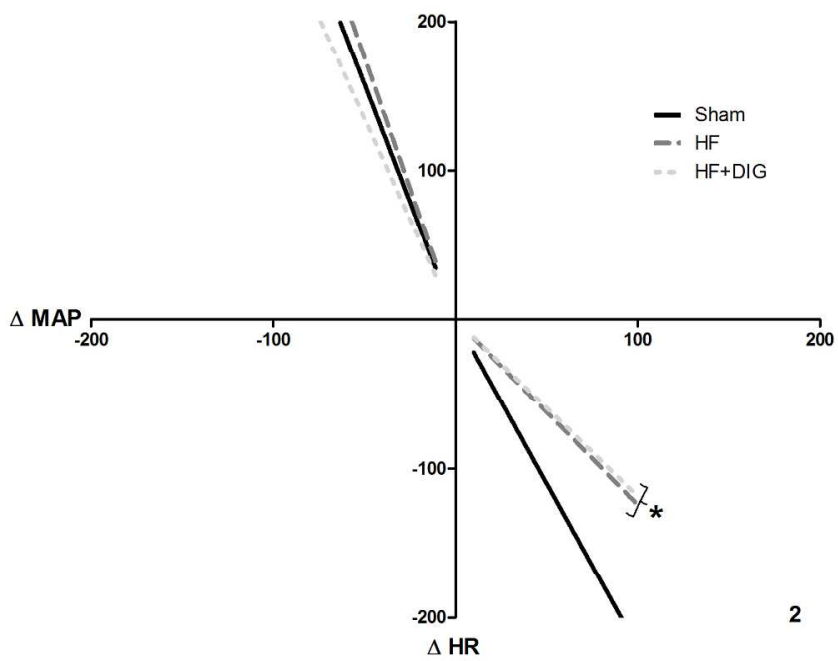

B

Baroreflex sensitivity of renal sympathe tic nerve activity

1

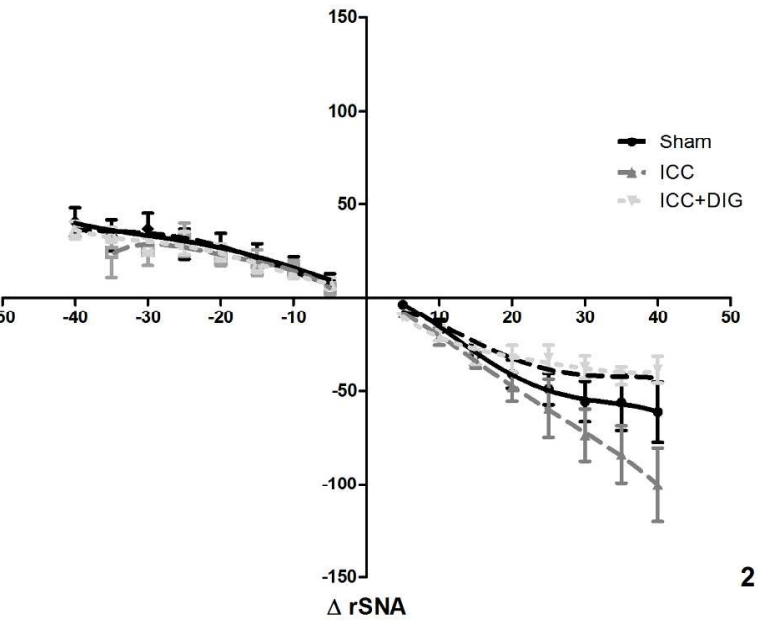

$161 \times 250 \mathrm{~mm}(300 \times 300 \mathrm{DPI})$

https://mc06.manuscriptcentral.com/cjpp-pubs 

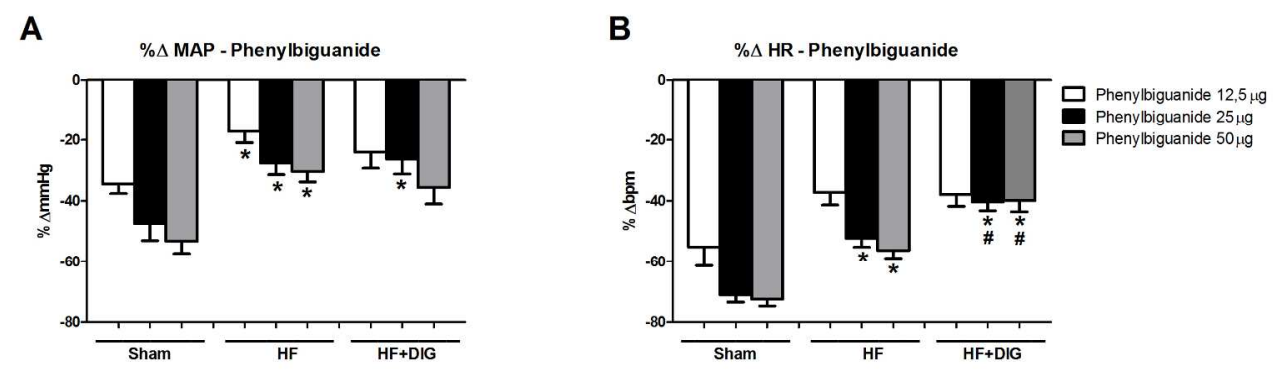

$222 \times 70 \mathrm{~mm}(300 \times 300 \mathrm{DPI})$ 


\begin{tabular}{lccc}
\hline \hline & \multicolumn{2}{c}{ Groups } & \\
\cline { 2 - 4 } \multicolumn{1}{c}{ Parameters } & $\begin{array}{c}\text { Sham } \\
(\mathbf{N}=\mathbf{5})\end{array}$ & $\begin{array}{c}\text { HF } \\
(\mathbf{N}=\mathbf{9})\end{array}$ & $\begin{array}{c}\text { HF+DIG } \\
(\mathbf{N}=\mathbf{5})\end{array}$ \\
\hline Myocardial Infarction Size & $0 \%$ & $47 \%$ & $47 \%$ \\
Pulmonary water content & $78,1 \pm 0,3 \%$ & $80,8 \pm 0,2 \%^{*}$ & $80,6 \pm 0,1 \% *$ \\
Fractional Shortening of the Transverse Area & $0,73 \pm 0,008 \%$ & $0,44 \pm 0,02 \% \%^{*}$ & $0,37 \pm 0,06 \%^{*}$ \\
Systolic area & $0,07 \pm 0,006 \mathrm{~cm}^{2}$ & $0,30 \pm 0,03 \mathrm{~cm}^{2 *}$ & $0,41 \pm 0,06 \mathrm{~cm}^{2 *}$ \\
Systolic circumference & $0,86 \pm 0,1 \mathrm{~cm}$ & $1,81 \pm 0,1 \mathrm{~cm}^{*}$ & $2,18 \pm 0,1 \mathrm{~cm}^{*}$ \\
\hline \hline
\end{tabular}

Table 1. Doppler echocardiographic parameters for the Sham, Heart Failure (HF) and HF treated with digitoxin $(H F+D I G)$ groups. Values are the mean \pm standard error of the mean. ${ }^{*} p<0.05$ compared with the Sham group. One-way ANOVA followed by Tukey post-test.

\begin{tabular}{|c|c|c|c|}
\hline & $\begin{array}{l}\text { Sham } \\
(\mathrm{N}=9)\end{array}$ & $\begin{array}{c}\text { HF } \\
(\mathrm{N}=8)\end{array}$ & $\begin{array}{c}\text { HF+DIG } \\
(\mathrm{N}=9)\end{array}$ \\
\hline \multicolumn{4}{|c|}{ Pulse Interval } \\
\hline $\operatorname{LFb}\left(\mathrm{ms}^{2}\right)$ & $14 \pm 3$ & $23 \pm 5^{*}$ & $15 \pm 3^{\#}$ \\
\hline $\mathrm{HFb}\left(\mathrm{ms}^{2}\right)$ & $86 \pm 3$ & $77 \pm 5^{*}$ & $85 \pm 3^{\#}$ \\
\hline LFb/HFb & $0.2 \pm 0.05$ & $0,4 \pm 0.2^{*}$ & $0.2 \pm 0.04^{\#}$ \\
\hline \multicolumn{4}{|c|}{ Systolic Blood Pressure } \\
\hline $\mathrm{LFb}\left(\mathrm{mmHg}{ }^{2}\right)$ & $5 \pm 2$ & $6 \pm 1$ & $2 \pm 0,3^{* \#}$ \\
\hline $\mathrm{HFb}\left(\mathrm{mmHg}^{2}\right)$ & $2 \pm 0.4$ & $3 \pm 0.4^{*}$ & $1 \pm 0.3^{* \#}$ \\
\hline
\end{tabular}

Table 2. Blood pressure and pulse interval variability for the Sham, Heart Failure (HF) and HF treated with digitoxin $(\mathrm{HF}+\mathrm{DIG})$ groups. Values were compared and are presented as the mean \pm standard deviation. ANOVA test followed by Tukey post-test. ${ }^{*} p<0.05$ vs Sham and $\# p<0.05$ vs HF. LFb $=$ Low frequency band, $\mathrm{HFb}=$ High frequency band. 


\begin{tabular}{lcc}
\hline \hline Groups & \multicolumn{2}{c}{ Reflex responses for heart rate } \\
& $\begin{array}{l}\text { Pressor response } \\
\text { Slope }(\mathrm{pps} / \mathrm{mmHg})\end{array}$ & $\begin{array}{c}\text { Depressor response } \\
\text { Slope }(\mathrm{pps} / \mathrm{mmHg})\end{array}$ \\
\hline Sham & $-2.27 \pm 0.23, \mathrm{~N}=6$ & $-3.17 \pm 0.32, \mathrm{~N}=6$ \\
HF & $-1.24 \pm 0.07, \mathrm{~N}=8^{*}$ & $-3.54 \pm 0.27, \mathrm{~N}=8$ \\
HF+DIG & $-1.18 \pm 0.09, \mathrm{~N}=9^{*}$ & $-2.86 \pm 0.25, \mathrm{~N}=9$ \\
& & \\
& Reflex responses of renal sympathetic nerve activity \\
Grupos & Pressor response & Depressor response \\
& Slope (pps/mmHg) & Slope(pps/mmHg) \\
\hline Sham & $-1.94 \pm 0.45, \mathrm{~N}=5$ & $-0.82 \pm 0.15, \mathrm{~N}=5$ \\
HF & $-2.62 \pm 0.30, \mathrm{~N}=7$ & $-0.81 \pm 0.35, \mathrm{~N}=5$ \\
HF+DIG & $-1.63 \pm 0.49, \mathrm{~N}=7$ & $-0.80 \pm 0.08, \mathrm{~N}=7$ \\
\hline \hline
\end{tabular}

Table 3. Slope values obtained from the linear regression line of the baroreceptor reflex function control for heart rate (HR) and renal sympathetic nerve activity (rSNA) for the Sham, Heart Failure (HF) and $\mathrm{HF}$ treated with digitoxin (HF+DIG) groups. Values are the mean \pm standard error of the mean. ${ }^{*} p<0.05$ compared with the Sham group. One-way ANOVA followed by Tukey post-test. 